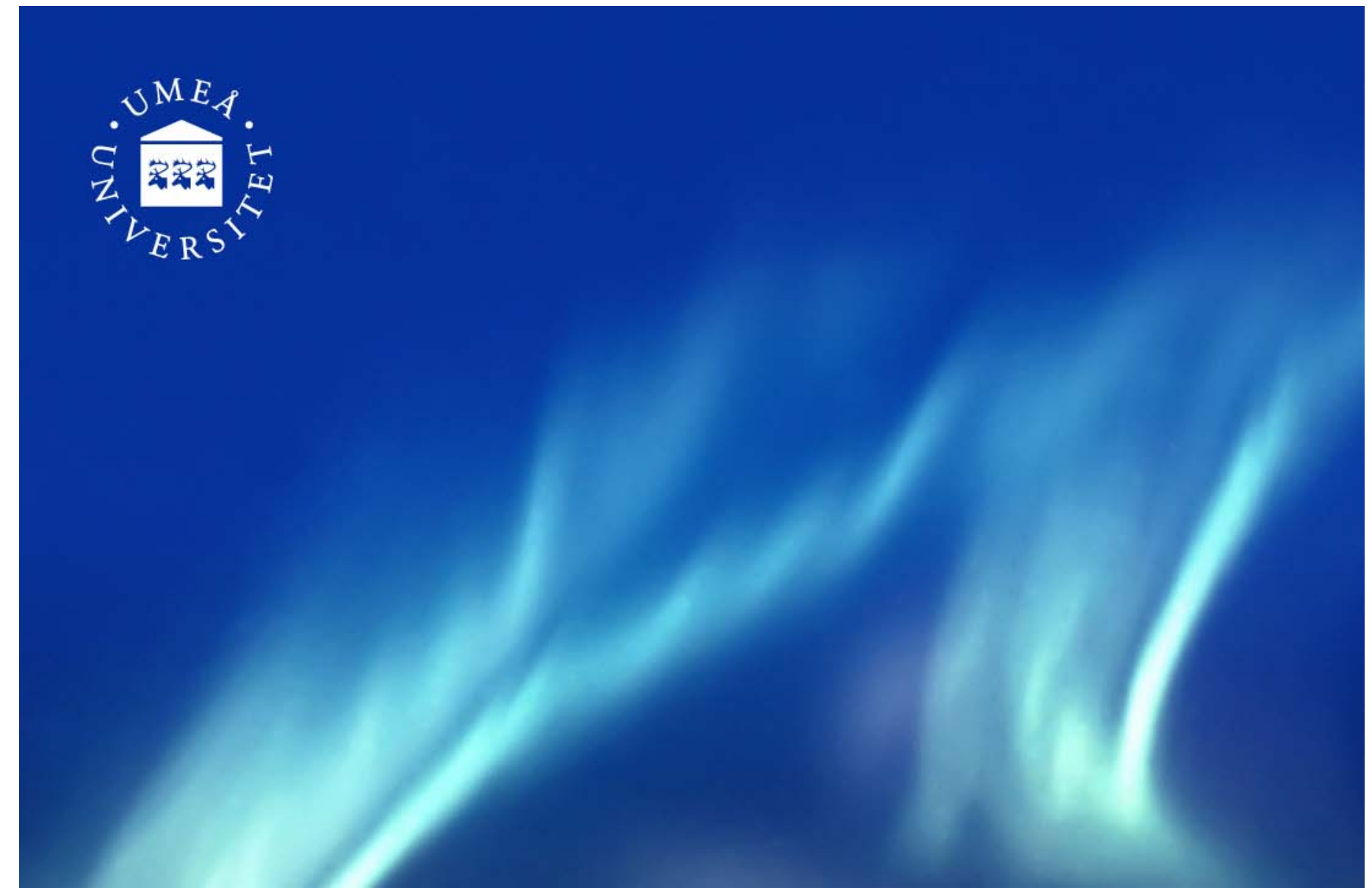

DiVA - Digitala Vetenskapliga Arkivet http://umu.diva-portal.org

This is an article published in Astrophysical Journal.

Citation for the published paper:

Kristof Stasiewicz; Jonas Ekeberg

Electric potentials and energy fluxes available for particle acceleration by alfvenons in the solar corona

Astrophysical Journal, 2008, Vol 680, Issue 2, L153-L156

DOI: $10.1086 / 589878$ 


\title{
ELECTRIC POTENTIALS AND ENERGY FLUXES AVAILABLE FOR PARTICLE ACCELERATION BY ALFVENONS IN THE SOLAR CORONA
}

\author{
K. STASIEWICZ ${ }^{1,2}$ AND J. EKEBERG ${ }^{1}$ \\ Received 2008 January 15; accepted 2008 May 6; published 2008 May 28
}

\begin{abstract}
We show that solitary wave solutions of the one- and two-fluid MHD equations, here called alfvenons, represent two types of electric field structures with negative or positive potentials that can explain the acceleration of particles in the solar corona. Negative potentials are created self-consistently by fast alfvenons and can reach hundreds of kilovolts, which could accelerate the electrons that produce X-ray emissions during flares. Slow alfvenons create positive potential structures of a few $\mathrm{kV}$ that accelerate solar wind ions. These alfvenons can be powered by irregular plasma flows in the photosphere and chromosphere, as well as by time-varying magnetic fields during reconnection events at the tops of coronal loops. Similar alfvenon structures are observed in the terrestrial magnetosphere, where they accelerate particles related to aurorae.
\end{abstract}

Subject headings: acceleration of particles — MHD — Sun: corona — Sun: flares — Sun: X-rays, gamma rays

\section{INTRODUCTION}

The generation of solar wind with protons streaming at 300$800 \mathrm{~km} \mathrm{~s}^{-1}$, after acquiring first the gravitational escape speed of $600 \mathrm{~km} \mathrm{~s}^{-1}$, implies the presence of positive acceleration potentials of $2-5 \mathrm{kV}$ in the corona. Scattering a fraction $(\sim 0.5$ $\mathrm{keV}$ ) of the ion kinetic energy would produce particles with high coronal temperatures: $\sim 5 \mathrm{MK}$. The active Sun emits Xrays corresponding to electron energies of tens to hundreds of $\mathrm{keV}$, which requires the presence of accelerating potentials of hundreds of $\mathrm{kV}$ in active regions. Furthermore, the energy spectrum of particles accelerated in the solar corona extends to tens of MeV (Miller 1998; Aschwanden 2004), suggesting the presence of correspondingly high effective potentials. Thus, the major problem of solar physics consists of finding explanations for the emergence of electric field structures with sufficiently high voltages, capable of accelerating solar wind ions to $\sim 3$ $\mathrm{kV}$ and electrons in flares to $\sim 100 \mathrm{kV}$. The highly publicized problem of "the heating of the corona" requires only the scattering of a small portion of the beam energy of solar wind ions or of accelerated electrons.

Recently, it has been demonstrated that structures with large electric potentials can be spontaneously created by nonlinear magnetohydrodynamic waves (alfvenons) in the solar corona (Stasiewicz 2006) and in the magnetosphere (Stasiewicz 2007). Although it is well known that one- or two-fluid MHD equations describe various branches of sinusoidal waves (Stringer 1963), it is less known that these equations also describe exponentially varying solutions that form solitons (McKenzie et al. 2004). Concepts of flare energy transport by Alfvén waves were invoked by many authors, and recently by Fletcher \& Hudson (2008). Large-amplitude nonlinear solitary structures are not theoretical speculations, but phenomena observed by the Cluster and other spacecraft at the bow shock, at the magnetosheath, and in the magnetosphere, which have been quantitatively modeled as solutions of two-fluid, or Hall-MHD, equations for collisionless plasmas (Stasiewicz 2004a, 2004b, 2005).

Three relevant questions arise: (1) What is the role of alfvenons in the generation of solar wind and in the acceleration of

\footnotetext{
${ }^{1}$ Swedish Institute of Space Physics, Uppsala and Kiruna, Sweden; k.stasiewicz@irfu.se, jonas.ekeberg@irf.se.

${ }^{2}$ Space Research Centre, Polish Academy of Sciences, Warsaw, Poland.
}

particles in flares? (2) What are the energy fluxes that can be transmitted along magnetic field lines by these nonlinear structures. (3) How large are the electric potentials available for acceleration of plasma in the solar corona? Answers to these questions are provided below.

\section{ELECTRIC FIELD AND POYNTING FLUX}

Consider an MHD wave in the plasma rest frame of reference, with speed $\omega / k$ in the $x$-direction at an angle $\alpha$ to the background magnetic field $\boldsymbol{B}_{0}=B_{0}(\cos \alpha, 0, \sin \alpha)$. We assume that plasma parameters vary mainly along $x$, so that $\partial / \partial x \gg \partial / \partial y, \partial / \partial z$, and transverse gradients can be neglected. Under such an assumption, the electric and magnetic fields are $\boldsymbol{E}=\left(\delta E_{x}, \delta E_{y}, \delta E_{z}\right)$ and $\boldsymbol{B}=\left(B_{x 0}, \delta B_{y}, B_{z 0}+\delta B_{z}\right)$, where delta quantities represent wave perturbations of arbitrary amplitude. The power available for acceleration of plasma by waves is related to the electromagnetic energy (Poynting) flux, $S=$ $\mu_{0}^{-1} \boldsymbol{E} \times \boldsymbol{B}$. The component of this flux along the $\boldsymbol{B}_{0}$ direction is given by

$$
\mu_{0} S_{\|}=\left(\sin \alpha \delta E_{x}-\cos \alpha \delta E_{z}\right) \delta B_{y}+\cos \alpha \delta B_{z} \delta E_{y},
$$

where $\delta B_{y}$ and $\delta B_{z}$ correspond to Alfvénic and magnetosonic polarizations, respectively. In the case of sinusoidal waves, $\propto \exp (i k x-i \omega t)$, the Maxwell's equation $\boldsymbol{\nabla} \times \boldsymbol{E}=-\partial \boldsymbol{B} / \partial t$ implies

$$
\delta E_{y}=(\omega / k) \delta B_{z}, \quad \delta E_{z}=-(\omega / k) \delta B_{y}
$$

while the $x$-component can be obtained from the generalized Ohm's law as

$$
\frac{\delta E_{x}}{V_{\mathrm{A}} B_{0}} \approx M \frac{\delta B_{y}}{B_{0}} \tan \alpha-\frac{\beta_{e} \lambda_{i}}{2 n} \frac{\partial}{\partial x}\left(\frac{p_{e}}{p_{e 0}}\right) .
$$

Here, $n=N / N_{0}, V_{\mathrm{A}}=B_{0}\left(\mu_{0} N_{0} m_{i}\right)^{-1 / 2}$ is the Alfvén speed, $M=\omega / k V_{\mathrm{A}}$ is the Alfvén Mach number, $p_{e}=p_{e 0} n^{\gamma}$ is the electron pressure, $\beta_{e}$ is the ratio of electron and magnetic field pressures, and $\lambda_{i}=V_{\mathrm{A}} / \omega_{c i}$ is the ion inertial length. A small term proportional to $m_{e} / m_{i}$, the electron to ion mass ratio, is neglected in equation (3). It is easily verified that the parallel electric field, $\boldsymbol{E} \cdot \boldsymbol{B} / B$, contains only the electron pressure term, 
consistent with equation (7). The Poynting flux in the plasma frame is then

$$
\frac{S_{\|}}{S_{\mathrm{A}}}=M_{\|} \frac{\delta B_{y}^{2}}{B_{0}^{2}}+M \cos \alpha \frac{\delta B_{z}^{2}}{B_{0}^{2}}-\sin \alpha \frac{\beta_{e} \lambda_{i}}{2 n} \frac{\partial}{\partial x}\left(\frac{p_{e}}{p_{e 0}}\right) \frac{\delta B_{y}}{B_{0}},
$$

in units of $S_{\mathrm{A}}=V_{\mathrm{A}} B_{0}^{2} / \mu_{0}$, with $M_{\|}=M / \cos \alpha$. Replacing magnetic perturbations by velocity perturbations as implied by the momentum equations, $\delta B_{y, z} B_{0}^{-1}=M_{\|} \delta V_{y, z} V_{\mathrm{A}}^{-1}$, we find an equivalent form

$$
\begin{aligned}
\frac{S_{\|}}{N_{0} m_{i} V_{\mathrm{A}}^{3}}= & M_{\|}^{3}\left(\frac{\delta V_{y}^{2}}{V_{\mathrm{A}}^{2}}+\cos ^{2} \alpha \frac{\delta V_{z}^{2}}{V_{\mathrm{A}}^{2}}\right) \\
& -M_{\|} \sin \alpha \frac{\beta_{e} \lambda_{i}}{2 n} \frac{\partial}{\partial x}\left(\frac{p_{e}}{p_{e 0}}\right) \frac{\delta V_{y}}{V_{\mathrm{A}}},
\end{aligned}
$$

which implies that there are ion flows $\delta V_{y}, \delta V_{z}$ that by shaking magnetic field lines $\delta B_{y}, \delta B_{z}$ create parallel Poynting flux $S_{\|}$. For a particular mode, one has to use a suitable Mach number: $M=\cos \alpha$ for Alfvén modes, $M=1$ for fast magnetosonic waves, $M=(\gamma \beta / 2)^{1 / 2} \cos \alpha$ for slow magnetosonic waves, and $M=(\gamma \beta / 2)^{1 / 2}$ for acoustic waves, with $\beta=\beta_{i}+\beta_{e}$.

\section{NONLINEAR WAVES AND ACCELERATION}

The location of linear and nonlinear waves in the phase space $(M, \alpha)$ is best studied by using a general dispersion equation for two-fluid waves (Stasiewicz 2007),

$$
k^{2} \lambda_{i}^{2}=\frac{A\left(A M_{\|}^{-2}-D\right)}{\left(m_{e} / m_{i}\right)\left(2 A-M_{\|}^{2} D\right)-1},
$$

where $A=M_{\|}^{2}-1$ and $D=\sin ^{2} \alpha /\left(M^{2}-\gamma \beta / 2\right)$. A quadratic term proportional to $\left(m_{e} / m_{i}\right)^{2}$ has been neglected. Note that when $k \lambda_{i}=0$, the factor $A=0$ describes nondispersive Alfvén waves, and the second factor, $A M_{\|}^{-2}-D=0$, the slow and fast magnetosonic waves. Equation (6) is applicable to Alfvén, kinetic Alfvén (KAW), inertial electron Alfvén (IEAW), magnetosonic, acoustic, lower hybrid (LH), and whistler waves, as visualized in Figure 1. Regions in the parameter space which give positive $k^{2} \lambda_{i}^{2}>0$ correspond to exponentially varying solutions (solitons; colored regions), while $k^{2} \lambda_{i}^{2}<0$ correspond to linear, sinusoidal solutions (white regions). Both types of solutions represent normal modes of the system, which exactly conserve the number flux, the momentum, and the energy flux.

The nonlinear equations listed by Stasiewicz $(2005,2007)$ are solved numerically in order to find the spatial profiles of $\delta B_{y}, \delta B_{z}, \delta E_{x}, \delta N$, which are used to determine the Poynting flux (eq. [1]) and integrated potential $\Phi=-\int \delta E_{x} d x$. Generally, these equilibrium solutions represent solitary, bipolar electric structures with either positive or negative potential. The sign of the potential is determined by the sign of $E_{x}$ in equation (3), which depends on the sense of polarization of the transverse magnetic field, or sign of $\delta B_{y}$. Left/right hand polarized structures correspond to slow/fast alfvenons and produce positive/ negative potentials.

The presence of a perpendicular potential implies the creation of a parallel potential difference of the same magnitude, and vice versa. The parallel potential is related to $E_{\|}$that can be

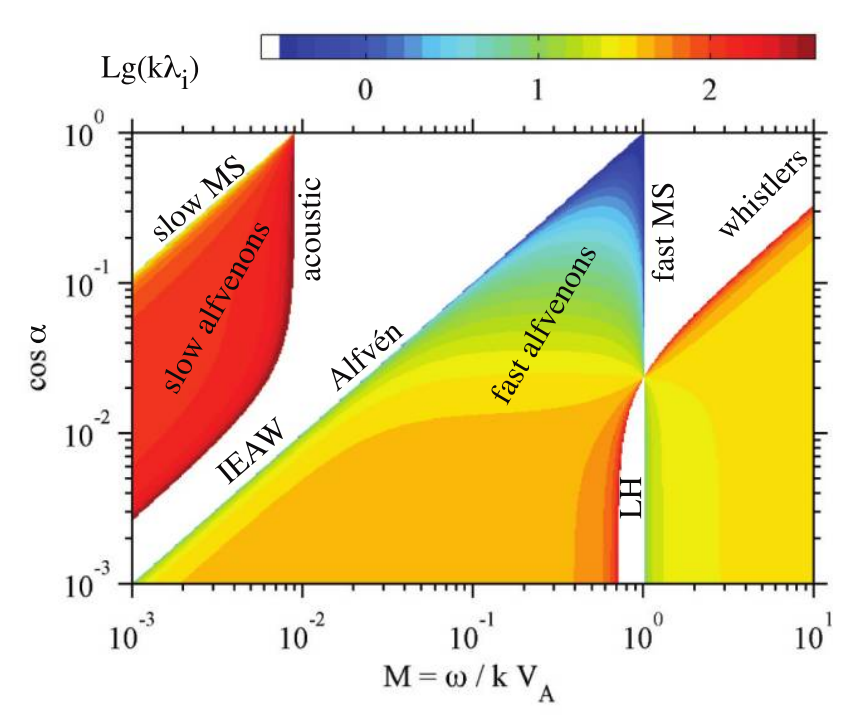

FIG. 1.-Phase-space diagram showing the spatial growth rate (eq. [6]) of nonlinear soliton solutions for a plasma with $\beta=10^{-4}, \gamma=5 / 3$. White areas correspond to sinusoidal solutions of linear wave modes including inertial electron Alfvén (IEAW) and lower hybrid (LH) waves.

obtained from the generalized Ohm's law (e.g., Krall \& Trivelpiece 1973) as

$$
\begin{gathered}
E_{\|}=\eta J_{\|}-\frac{\nabla_{\|} p_{\| e}}{N e}-\frac{\left(p_{\perp e}-p_{\| e}\right)}{N e} \frac{(\boldsymbol{\nabla} B)_{\|}}{B} \\
+\frac{m_{e}}{N e^{2}}\left[\boldsymbol{\nabla} \cdot\left(\boldsymbol{V} J_{\|}+\boldsymbol{J} V_{\|}\right)+\frac{\partial J_{\|}}{\partial t}\right] .
\end{gathered}
$$

Thus, there are four mechanisms supporting $E_{\|}$in two-fluid MHD: (1) classical or anomalous resistivity, $\eta$; (2) the electron pressure gradient along the magnetic field; (3) the magnetic mirror force acting on anisotropic electron pressures; and (4) electron inertial effects. The above equation is valid for all wave modes and structures in fluid approach. Yet another mechanism for the parallel electric field can be provided by double layers (e.g., Charles 2007) which are on smaller, Debye length scales and require kinetic description. Phenomenologically, they can be included in equation (7) as a sort of anomalous resistivity.

The electric potential $\Phi$ modifies particles trajectories in the phase space. Conservation of the total energy, $m v_{\perp}^{2} / 2+$ $m v_{\|}^{2} / 2+q \Phi+m \Phi_{G}=$ const, where $\Phi_{G}$ is the gravitational potential, implies that particles entering the structure would change their kinetic energy by an amount corresponding to the difference of the potential energies. In a collisionless plasma the perpendicular velocity is constrained by conservation of the first adiabatic invariant, leading to an increase of $v_{\|}^{2}$ and lowering of the mirroring altitude of particles to denser regions where they can be subject to collisions. Particles will dissipate kinetic energy by instabilities related to pitch angle anisotropy, through X-ray emissions, heating of the ambient plasma, and ionization of the neutral atmosphere at low altitudes. Heating can be also accomplished by ion Landau damping of slow modes and electron Landau damping of fast modes. The dissipative processes are beyond the formalism of the present stationary model and their description would require a fully kinetic model supported by simulations. 


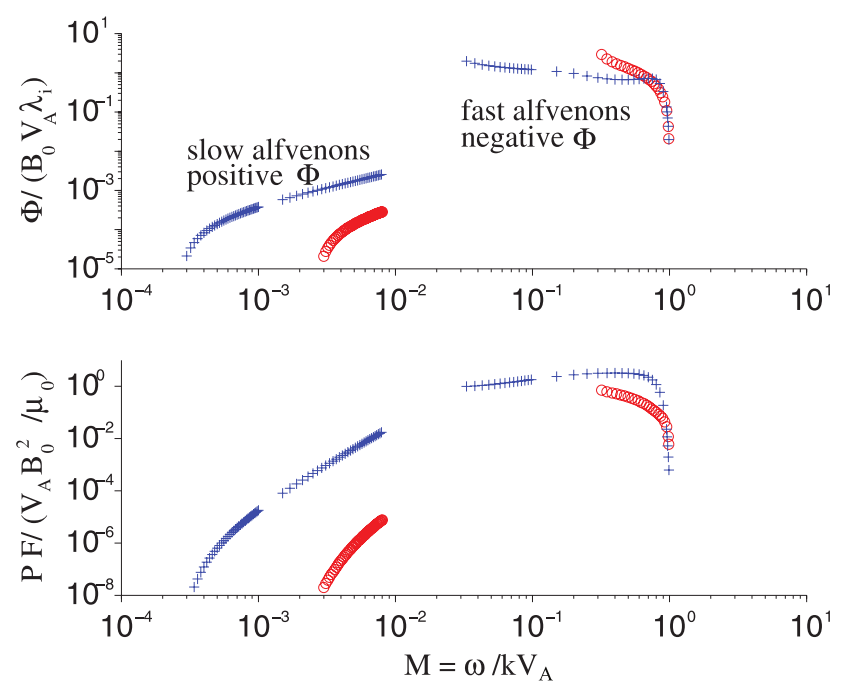

FIG. 2.-Maximum electric potential in units of $\Phi_{\mathrm{A}}=V_{\mathrm{A}} B_{0} \lambda_{i}$ (top) and maximum Poynting flux in units of $S_{\mathrm{A}}=\mu_{0}^{-1} V_{\mathrm{A}} B_{0}^{2}$ (bottom) for solitons propagating at $\cos \alpha=0.03$ (blue plus signs) and $\cos \alpha=0.3$ (red circles) in a plasma with $\beta=10^{-4}$ and $\gamma=5 / 3$.

\section{FAST ALFVENONS AND SOLAR FLARES}

Each point in Figure 1 represents a solution which uniquely determines the size of the soliton (here called alfvenon) and its physical properties such as the electric field, potential, magnetic field, currents, and density perturbations. Values of maximum electric potential and Poynting flux for alfvenons propagating at two different angles, $\cos \alpha=0.03$ and $\cos \alpha=$ 0.3 , have been extracted from computed profiles and are shown in Figure 2. It can be seen that fast alfvenons located between the Alfvén mode and the fast magnetosonic mode can generate potentials that reach values $\Phi_{\mathrm{A}}=B_{0} V_{\mathrm{A}} \lambda_{i}$ (shown in Table 1 , assuming a proton plasma). This potential decreases when approaching the fast mode $M=1$. On the other hand, slow alfvenons, located between the IEAW/acoustic and the slow magnetosonic mode can produce potentials only up to $\sim 10^{-3} \Phi_{\mathrm{A}}$, which also become very small when approaching the slow mode. Figure 2 and Table 2 can be used to infer possible energy fluxes that could be carried by these alfvenons.

In Figure 2 and Table 1 we see that fast alfvenons in a plasma at, for example, $B=100 \mathrm{G}, N=10^{9} \mathrm{~cm}^{-3}$ can create electric potentials of $\Phi=-500 \mathrm{kV}$, but potentials of tens MV are also possible. It is natural to associate these alfvenons with solar flare events. The required Poynting flux could be produced both by irregular plasma flows in the chromosphere, equation (5), and by time varying magnetic fields, equation (4), during reconnection events at the top of coronal loops. Fast alfvenons create negative potentials, which can accelerate electrons in one direction, and ions in the opposite direction. Note that lower plasma densities create higher potentials, favoring high-altitude sources of the most energetic solar flares. In astrophysical objects with, for example, $B \sim 1 \mathrm{kG}$ and $N \sim 10 \mathrm{~cm}^{-3}$, fast alfvenons could create potentials of $\Phi_{\mathrm{A}} \sim 10^{15} \mathrm{~V}$, which may be relevant in the context of cosmic-ray generation.

Acceleration by coherent electric field structures implies that ion and electron footprints should not be colocated. Spatial separation of emissions from accelerated electrons and ions has recently been confirmed in RHESSI measurements by Hurford et al. (2003). Similar structures and processes are observed in
TABLE 1

Electric Potential $\Phi_{\mathrm{A}}=B_{0} V_{\mathrm{A}} \lambda_{i}(\mathrm{kV})$

\begin{tabular}{lrrll}
\hline \hline & \multicolumn{5}{c}{$B_{0}$} \\
\cline { 2 - 5 }$N\left(\mathrm{~cm}^{-3}\right)$ & $1000 \mathrm{G}$ & $100 \mathrm{G}$ & $10 \mathrm{G}$ & $1 \mathrm{G}$ \\
\hline $10^{11} \ldots \ldots$ & 500 & 5 & 0.05 & $\ldots$ \\
$10^{10} \ldots \ldots$ & 5000 & 50 & 0.5 & 0.005 \\
$10^{09} \ldots \ldots$ & 50000 & 500 & 5 & 0.05 \\
$10^{08} \ldots \ldots$ & \multicolumn{1}{c}{$\cdots$} & 5000 & 50 & 0.50 \\
\hline
\end{tabular}

the terrestrial magnetosphere, where earthward accelerated electrons (1-20 keV) producing aurorae are measured together with accelerated ion beams moving in the opposite direction (e.g., McFadden et al. 1999). Fast alfvenons represent a twist of magnetic field lines, where the transverse components $\delta B_{y}$ and $\delta B_{z}$ make helical rotations around the guide field. Because the structure propagates obliquely across the magnetic field it has continuous access to flux tubes with nondepleted electrons. Furthermore, some alfvenon solutions contain return fieldaligned currents, and they are very thin structures surrounded by ambient plasma. This could provide a mechanism for recycling electrons involved in parallel acceleration, solving possibly the problem of number of electrons needed to maintain long-duration flare activity (Fletcher \& Hudson 2008).

\section{SLOW ALFVENONS AND THE SOLAR WIND}

For the acceleration of the solar wind, much smaller potentials, $2-5 \mathrm{kV}$, are needed. These potentials should be positive in order to drive ions out from the chromosphere. A natural candidate for such electric structures are the slow alfvenons shown in Figure 2. The slow alfvenons create positive potentials of $10^{-3} \Phi_{\mathrm{A}}$. A combination of $B, N$ parameters that give $\Phi_{\mathrm{A}} \sim 5000 \mathrm{kV}$ in Table 1 would support electric field structures with a potential corresponding to the typical energy of solar wind ions. The Poynting flux (eq. [5]) driving slow alfvenons is most likely created by irregular plasma flows related to the universal convection granulation observed in the Sun's photosphere.

A connection between slow mode waves and sources of solar wind has recently been confirmed with Hinode data by Cirtain et al. (2007) and Sakao et al. (2007). The presence of slowmode structures on coronal loops has also been confirmed experimentally by De Moortel et al. (2000) and Robbrecht et al. (2001). The present model appears to be consistent also with observations (e.g., Woo \& Habbal 2005), which indicate that slow wind is initially confined in closed loops at borders between active regions and coronal holes, while the fast wind is cooler, less dense, and emanates directly from coronal holes with steeper radial density gradients. Indeed, Table 1 shows that less dense plasma would support larger potentials, provided the magnetic field strengths are comparable in both cases.

TABLE 2

ENERGY FLUX $S_{\mathrm{A}}=\mu_{0}^{-1} V_{\mathrm{A}} B_{0}^{2}\left(\mathrm{~W} \mathrm{~m}^{-2}\right)$

\begin{tabular}{|c|c|c|c|c|}
\hline \multirow[b]{2}{*}{$N\left(\mathrm{~cm}^{-3}\right)$} & \multicolumn{4}{|c|}{$B_{0}$} \\
\hline & $1000 \mathrm{G}$ & $100 \mathrm{G}$ & $10 \mathrm{G}$ & $1 \mathrm{G}$ \\
\hline $10^{11}$ & $5.5 \mathrm{E}+10$ & $5.5 \mathrm{E}+7$ & $5.5 \mathrm{E}+4$ & \\
\hline $10^{10}$ & $1.7 \mathrm{E}+11$ & $1.7 \mathrm{E}+8$ & $1.7 E+5$ & $1.7 \mathrm{E}+2$ \\
\hline $10^{09}$ & $5.5 \mathrm{E}+11$ & $5.5 \mathrm{E}+8$ & $5.5 \mathrm{E}+5$ & $5.5 \mathrm{E}+2$ \\
\hline $10^{08} \ldots$ & $\ldots$ & $1.7 \mathrm{E}+9$ & $1.7 \mathrm{E}+6$ & $1.7 \mathrm{E}+3$ \\
\hline
\end{tabular}




\section{DISCUSSION}

The generic mechanism for parallel Poynting flux generation by magnetic footprint motions described by equation (5) would lead to a gradual buildup of magnetic and thermal energies of the loop until the system becomes unstable and ends up with eruptions such as flares or coronal mass ejections. Alfvenons carry large field-aligned currents, so they would contribute to the magnetic structure of coronal loops. The acceleration of particles by coherent electric field structures represents a onestep process, different from stochastic acceleration (e.g., Petrosian \& Liu 2004), which is described by quasi-linear diffusion equation. However, particles trapped on closed loops could have multiple interactions with alfvenons and be accelerated to higher energies. Loop-top regions of hard X-ray emissions identified in Yohkoh data by Masuda et al. (1994) could correspond to such trapped particles accelerated by alfvenons.

The scale of alfvenons propagating quasi-perpendicularly is typically several ion inertial lengths (Stasiewicz \& Ekeberg 2007), a tiny scale in the corona, where $\lambda_{i}=7 \mathrm{~m}$ for $N=$ $10^{9} \mathrm{~cm}^{-3}$. The parallel scale depends on the propagation angle, $L_{\|}=L_{\perp} \tan \alpha$, and for $\alpha=90^{\circ}$ it corresponds to global fieldline oscillations (e.g., Nakariakov 2007). An alfvenon with $L_{\perp} \sim 10 \lambda_{i} \sim 100 \mathrm{~m}$ propagating at $\alpha=89.94^{\circ}$ would have length $L_{\|} \approx 100 \mathrm{~km}$, which corresponds to the size of elementary acceleration structures inferred from high time resolution measurements of radio emissions generated by accelerated electron beams (Xie et al. 2000). Observations of drifting frequency patterns are commonly interpreted as related to electron beams produced in the reconnection region (Aschwanden \& Benz 1997). However, bidirectional electron beams generating strong Langmuir waves $\left(\sim 1 \mathrm{~V} \mathrm{~m}^{-1}\right)$ are associated with alfvenons observed in auroral regions (e.g., Stasiewicz et al. 1997 [see Figs. 11-13]).

X-ray structures in the solar corona are likely caused by similar acceleration processes as auroral arcs, with appropriate scaling of thickness by $\lambda_{i}$ and potentials by $\Phi_{\mathrm{A}}$ (Stasiewicz 2006, 2007). The large-scale auroral structures have thickness $L_{\perp} \sim \lambda_{i} \sim 100 \mathrm{~km}$ and may extend horizontally to thousands of $\mathrm{km}$ in the $y$-direction. Because of the scaling properties, $\lambda_{i} \propto N^{-1 / 2}$, the alfvenons in the solar corona at $N \sim 10^{8}-10^{9}$ $\mathrm{cm}^{-3}$ are expected to be $10^{3}-10^{4}$ times thinner than the corresponding structures measured in the magnetosphere at $N \sim$ $10^{1}-10^{2} \mathrm{~cm}^{-3}$. Negative and positive potential structures are observed by many magnetospheric satellites in the auroral regions (e.g., Ergun et al. 1998), including the recent Cluster mission (Marklund et al. 2001).

The transverse horizontal extension of alfvenons ( $y$-direction) can match the driver size of solar convection granulation, and the accelerated plasma will spread along $\boldsymbol{B}$; both dimensions are of many $\mathrm{Mm}$, which should be detectable by optical instruments. Thin threads and arcades of heated plasma seen in images from the TRACE spacecraft ${ }^{3}$ and Hinode (Cirtain et al. 2007) are possibly manifestations of remnants of alfvenons described above.

\section{CONCLUSIONS}

We have shown that the acceleration of the solar wind to the observed potentials $2-5 \mathrm{kV}$ can be accomplished by electric structures of slow alfvenons driven by irregular plasma flows in the photospheric granulation. Slow alfvenons create positive potentials expelling ions into interplanetary space. Transferring some $15 \%$ of the ion energy to the electrons by collisions or plasma instabilities, viz., slowing down of the beam ions, could explain heating of the corona to $\sim 5 \mathrm{MK}$. Acceleration of particles in solar flares and additional electron heating can be attributed to fast alfvenons created by varying magnetic fields in coronal loops. Fast alfvenons are shown to support negative potentials of hundreds of $\mathrm{kV}$, providing an explanation for the energies of X-rays and gamma rays created in the solar corona during flares. Similar structures (with sizes scaled by $\lambda_{i}$ and potentials by $\Phi_{\mathrm{A}}=B_{0} V_{\mathrm{A}} \lambda_{i}$ ) are observed in the terrestrial magnetosphere, which provides a strong argument in favor of their existence also in the solar corona and a motivation for further development of the present model.

J. Ekeberg is financed by the Swedish National Graduate School of Space Technology.

${ }^{3}$ See http://trace.lmsal.com.

\section{REFERENCES}

Aschwanden, M. 2004, Physics of the Solar Corona (Berlin: Springer) Aschwanden, M. J., \& Benz, A. O. 1997, ApJ, 480, 825

Charles, C. 2007, Plasma Sources Sci. Technol., 16, R1

Cirtain, J. W., et al. 2007, Science, 318, 1580

Ergun, R. E., et al. 1998, Geophys. Res. Lett., 25, 2025

Fletcher, L., \& Hudson, H. S. 2008, ApJ, 675, 1645

Hurford, G. J., Schwartz, R. A., Krucker, S., Lin, R. P., Smith, D. M., \& Vilmer, N. 2003, ApJ, 595, L77

Krall, N. A., \& Trivelpiece, A. W. 1973, Principles of Plasma Physics (New York: McGraw-Hill), chap. 3

Marklund, G. T., et al. 2001, Nature, 414, 724

Masuda, S., Kosugi, T., Hara, H., Tsuneta, S., \& Ogawara, Y. 1994, Nature, 371,495

McFadden, J. P., Carlson, C. W., \& Ergun, R. E. 1999, J. Geophys. Res., 104, 14453

McKenzie, J. F., Dubinin, E., Sauer, K., \& Doyle, T. B. 2004, J. Plasma Phys., 70,431

Miller, J. A. 1998, Space Sci. Rev., 86, 79
De Moortel, I., Ireland, J., \& Walsh, R. W. 2000, A\&A, 355, L23

Nakariakov, V. M. 2007, Adv. Space Res., 39, 1804

Petrosian, V., \& Liu, S. M. 2004, ApJ, 610, 550

Robbrecht, E., Verwichte, E., Berghamans, D., Hochedez, J. F., Poedts, S., \& Nakariakov, V. M. 2001, A\&A, 370, 591

Sakao, T., et al. 2007, Science, 318, 1585

Stasiewicz, K. 2004a, Geophys. Res. Lett., 31, L21804

- 2004b, Phys. Rev. Lett., 93, 125004

. 2005, J. Geophys. Res., 110, A03220

2006, Phys. Rev. Lett., 96, 175003

2007, Plasma Phys. Controlled Fusion, 49, B621

Stasiewicz, K., \& Ekeberg, J. 2007, Phys. Rev. Lett., 99, 089502

Stasiewicz, K., Gustafsson, G., Marklund, G., Lindqvist, P.-A., Clemmons, J.

H., \& Zanetti, L. 1997, J. Geophys. Res., 102, 2565

Stringer, T. E. 1963, Plasma Phys., 5, 89

Woo, R., \& Habbal, S. R. 2005, ApJ, 629, L129

Xie, R. X., Fu, Q. J., Wang, M., \& Liu, Y. Y. 2000, Sol. Phys., 197, 375 